\title{
ON THE OSCULATING QUARTIC OF A PLANE CURVE*
}

\author{
BY \\ WILLIAM WELLS DENTON
}

The quartic curve which has contact of the thirteenth order with a given analytic plane curve at one of its points will be called the osculating quartic. In this paper, its equation will be found in an invariant form, referred to a triangle which has a simple projective relation to the given curve. The method which we shall employ is due to Professor Wilczrnskr. $\dagger$

Let there be given three linearly independent analytic functions of $x$,

$$
y_{\kappa}=f_{\kappa}(x), \quad(\kappa=1,2,3) .
$$

They may be interpreted as the homogeneous coördinates of a point $P_{v}$ in a plane. As $x$ changes, $P_{y}$ describes an analytic plane curve $C_{v}$. There exists a uniquely defined linear differential equation of the third order of which $y_{1}, y_{2}, y_{3}$ form a fundamental system. Let this be

$$
y^{(3)}+p_{1} y^{\prime \prime}+p_{2} y^{\prime}+p_{3} y=0,
$$

where $p_{1}, p_{2}, p_{3}$ are analytic functions of $x$. We may, therefore, speak of $C_{v}$ as being an integral curve of (2). But this integral curve is not unique, for every projective transformation of $C_{\nu}$ is likewise an integral curve of (2). Conversely, every integral curve of (2) is a projective transformation of $C_{y}$. Hence the properties of $C_{y}$ determined by the coefficients (2) are common to all curves projectively equivalent to $C_{y}$, that is, they are projective properties.

The representation of a curve in the form (1), however, involves some arbitrary elements. In the first place, since the coördinates are homogeneous, a transformation of the form

$$
\bar{y}=\lambda(x) y,
$$

where $\lambda(x)$ is an arbitrary analytic function of $x$, does rot affect the curve. Moreover, a transformation of the form

$$
\bar{x}=\xi(x),
$$

where $\xi(x)$ is an arbitrary analytic function of $x$, merely changes the para-

* Presented to the Society (Chicago), January 2, 1909.

†E. J. Wilczynski, Projective differential geometry of curves and ruled surfaces. Teubner, Leipzig, 1906, Chapters II and III. We shall hereafter refer to this work as Proj. Diff. Geom. 297 
metric representation, without changing the curve. Those combinations of the coefficients of (2) and of their derivatives which remain unchanged under all transformations of the form (3) and (4), the so-called invariants, therefore characterize the projective properties of the curve independently of its method of representation. If such an invariant function contains also $y$ and its derivatives it is called a covariant.

It is convenient to consider the subgroup of the general group of transformations defined by equations (3) and (4), which is obtained by leaving the indopendent variable $x$ fixed. The invariant functions for this subgroup are known as seminvariants and semicovariants. Of these we shall need *

$$
\begin{aligned}
z & =y^{\prime}+p_{1} y, & \rho & =y^{\prime \prime}+2 p_{1} y^{\prime}+p_{2} y, \\
\mathcal{B}_{2} & =p_{2}-p_{1}^{2}-p_{1}^{\prime}, & P_{3} & =p_{3}-3 p_{1} p_{2}+2 p_{1}^{3}-p_{1}^{\prime \prime},
\end{aligned}
$$

as ell as the following invariants $: \dagger$

$$
\theta_{3}=P_{3}-\frac{8}{2} P_{2}^{\prime}, \quad \theta_{8}=6 \theta_{3} \theta_{3}^{\prime \prime}-7\left(\theta_{3}^{\prime}\right)^{2}-27 P_{2} \theta_{3}^{2} .
$$

By a special transformation of the form (3) and (4), viz., $\ddagger$

$$
\bar{y}=\frac{d \bar{x}}{d x} y, \quad \bar{x}=\xi(x),
$$

where $\xi(x)$ is determined by the equations:

$$
\xi(x)=c_{1} \int e^{\int \eta d x} d x+c_{2}, \quad \eta^{\prime}-\frac{1}{2} \eta^{2}=\frac{3}{2} P_{2},
$$

in which $c_{1}$ and $c_{2}$ are arbitrary constants, equation (2) may be reduced to the LAGUERRE-FORSYTH canonical form :

$$
\frac{d^{3} \bar{y}}{d \bar{x}^{3}}+\bar{P}_{3} \bar{y}=0 \text {. }
$$

The invariant functions above mentioned have then the following exceptionally imple canonical forms :

$$
\begin{gathered}
\bar{z}=y^{\prime}, \quad \bar{\rho}=y^{\prime \prime}, \quad \bar{P}_{2}=0, \\
\bar{\theta}_{3}=\bar{P}_{3}, \quad \bar{\theta}_{8}=6 \bar{P}_{3} \bar{P}_{3}^{\prime \prime}-7\left(\bar{P}_{3}^{\prime}\right)^{2} .
\end{gathered}
$$

Hereafter, we shall assume the differential equation in this form, and, for convenience in writing, omit the dashes. We shall assume also that $P_{3}$, in this equation, is not identically equal to zero. This requires only that $C_{y}$ be not a conic.

* Proj. Liff. Geom., p. 58.

† Ibid., p. 59.

t Ibid., p. 25. 
Let $x_{0}$ be the value of $x$ which determines the point $P_{y_{0}}$, on the curve $C_{y}$. The point $P$, whose coördinates are

$$
z_{\kappa}=\left[\frac{d y_{\kappa}}{d x}\right]_{x=x_{0}} \quad(\kappa=1,2,3),
$$

is a point on the tangent to $C_{y}$ at $P_{y_{0}}$; $*$ and, if $P_{y_{0}}$ is not a point of inflection, the point $P_{\rho}$, whose coördinates are

$$
\rho_{\kappa}=\left[\frac{d^{2}}{d x} y_{\kappa=x_{0}}\right.
$$$$
(\kappa=1,2,3) \text {, }
$$

is not collinear with $P_{y_{0}}$ and $P_{s}$; so that these points determine a non-degenerate triangle, semicovariantly related to the curve $C_{y}$. Let this be taken as a triangle of reference. We may choose the unit point of our system of homogeneous coördinates so that an expression of the form $\dagger$

$$
x_{1} y\left(x_{0}\right)+x_{2} z\left(x_{0}\right)+x_{3} \rho\left(x_{0}\right)
$$

will represent the point whose coördinates are precisely $x_{1}, x_{2}, x_{3}$.

Let $x=x_{0}$ be an ordinary point for the function $P_{3}$; and, for convenience in writing, let $x_{0}=0$, since this assumption involves no loss of generality. Then for values of $|x|$ sufficiently small, any solution of equation (10) may be expressed as a convergent power series,

$$
G(x)=y(0)+y^{\prime}(0) x+\frac{1}{2} y^{\prime \prime}(0) x^{2}+\cdots+\frac{1}{1+!} y^{(14)}(0) x^{14}+\cdots
$$

From equation (10), we find by direct differentiation :

$$
\begin{aligned}
& y^{(3)}=-a y, \quad y^{(4)}=-a_{1} y-a z, \\
& y^{(5)}=-a_{2} y-2 a_{1} z-a \rho, \\
& y^{(6)}=-\left(a_{3}-a^{2}\right) y-3 a_{2} z-3 a_{1} \rho, \text { etc., }
\end{aligned}
$$

where we have put $y=y(0), z=y^{\prime}(0), \rho=y^{\prime \prime}(0), a=P_{3}$, and where $a_{m}^{n}$ is an abbreviation for the $n$th power of the $m$ th derivative of $P_{3}$ with respect to $x$. Putting $G(x)$ in the form (13), we find the following equations, which represent the curve $C_{y}$ up to terms of the fourteenth order in the vicinity of the point $P_{y_{0}}$ :

$$
\begin{aligned}
y_{1}= & -\frac{a}{3 !} x^{3}-\frac{a_{1}}{4 !} x^{4}-\frac{a_{2}}{5 !} x^{5}-\frac{1}{6 !}\left(a_{3}-a^{2}\right) x^{6}-\frac{1}{7 !}\left(a_{4}-5 a a_{1}\right) x^{7} \\
& -\frac{1}{8 !}\left(a_{5}-11 a a_{2}-5 a_{1}^{2}\right) x^{8}-\frac{1}{9 !}\left(a_{6}-21 a a_{3}-21 a_{1} a_{2}+a^{3}\right) x^{9}
\end{aligned}
$$

* Proj. Diff. Geom., p. 54.

† Proj. Diff. Geom., p. 61. 


$$
\begin{aligned}
& -\frac{1}{10 !}\left(a_{7}-36 a a_{4}-12 a_{1} a_{3}-21 a_{2}^{2}+12 a^{2} a_{1}\right) x^{10} \\
& -\frac{1}{11 !}\left(a_{8}-57 a a_{5}-78 a_{1} a_{4}-84 a_{2} a_{3}+39 a_{2} a^{2}+45 a a_{1}^{2}\right) x^{11} \\
& -\frac{1}{12 !}\left(a_{9}-85 a a_{6}-135 a_{1} a_{5}-162 a_{2} a_{4}+105 a^{2} a_{3}-84 a_{3}^{2}\right. \\
& \left.+300 a a_{1} a_{2}+45 a_{1}^{3}-a^{4}\right) x^{12} \\
& -\frac{1}{13 !}\left(a_{10}-220 a_{1} a_{6}-121 a a_{7}-297 a_{2} a_{5}-330 a_{3} a_{4}+852 a a_{1} a_{3}\right. \\
& \left.+246 a^{2} a_{4}+435 a_{1}^{2} a_{2}+516 a a_{2}^{2}-22 a^{3} a_{1}\right) x^{13} \\
& -\frac{1}{14 !}\left(a_{11}-166 a a_{8}-341 a_{1} a_{7}-517 a_{2} a_{6}-627 a_{3} a_{5}+519 a^{2} a_{5}\right. \\
& -330 a_{4}^{2}+2124 a a_{1} a_{4}+1287 a_{1}^{2} a_{3}+3054 a a_{2} a_{3} \\
& \left.+1386 a_{1} a_{2}^{2}-94 a^{3} a_{2}-177 a^{2} a_{1}^{2}\right) a^{14} \\
& y_{2}=x-\frac{a}{4 !} x^{4}-\frac{2 a_{1}}{5 !} x^{5}-\frac{3 a_{2}}{6 !} x^{6}-\frac{1}{7 ! !}\left(4 a_{3}-a^{2}\right) x^{7}-\frac{1}{8 !}\left(5 a_{4}-7 a a_{1}\right) x^{8} \\
& -\frac{1}{9 !}\left(6 a_{5}-18 a a_{2}-12 a_{1}^{2}\right) x^{9}-\frac{1}{10 !}\left(7 a_{6}+a^{3}-39 a a_{3}-63 a_{1} a_{2}\right) x^{10} \\
& -\frac{1}{11 !}\left(8 a_{7}-75 a a_{4}-144 a_{1} a_{3}-84 a_{2}^{2}+15 a^{2} a_{1}\right) x^{11} \\
& -\frac{1}{12 !}\left(9 a_{8}-132 a a_{5}-297 a_{1} a_{4}-396 a_{2} a_{3}+54 a^{2} a_{2}+75 a a_{1}^{2}\right) x^{12} \\
& -\frac{1}{13 !}\left(10 a_{9}-217 a a_{6}-564 a_{1} a_{5}-855 a_{2} a_{4}+159 a^{2} a_{3}-480 a_{3}^{2}\right. \\
& \left.+558 a a_{1} a_{2}+120 a_{1}^{3}-a^{4}\right) x^{13} \\
& -\frac{1}{14 !}\left(11 a_{10}-338 a a_{7}-1001 a_{1} a_{6}-1716 a_{2} a_{5}-2145 a_{3} a_{4}+405 a^{2} a_{4}\right. \\
& \left.+1728 a a_{1} a_{3}+1353 a_{1}^{2} a_{2}+1074 a a_{2}^{2}-26 a^{3} a_{1}\right) x^{14} \\
& -\cdots, \\
& y_{3}=\frac{1}{2} x^{2}-\frac{a}{5 !} x^{5}-\frac{3 a_{1}}{6 !} x^{6}-\frac{v a_{2}}{7 !} x^{7}-\frac{1}{8 !}\left(10 a_{3}-a^{2}\right) x^{3} \\
& -\frac{1}{9 !}\left(15 a_{1}-9 a a_{1}\right) x^{9}-\frac{1}{10 !}\left(21 a_{3}-27 a a_{2}-21 a_{1}^{2}\right) x^{10} \\
& -\frac{1}{11 !}\left(28 a_{6}-66 a a_{3}-132 a_{1} a_{2}+a^{3}\right) x^{11}
\end{aligned}
$$




$$
\begin{aligned}
& -\frac{1}{12 !}\left(36 a_{7}-141 a a_{4}-342 a_{1} a_{3}-216 a_{2}^{2}+18 a^{2} a_{1}\right) x^{12} \\
& -\frac{1}{13 !}\left(45 a_{8}-273 a a_{5}-780 a_{1} a_{4}-1170 a_{2} a_{3}+72 a_{2} a^{2}+111 a a_{1}^{2}\right) x^{13} \\
& -\frac{1}{14 !}\left(55 a_{9}-490 a a_{6}-1617 a_{1} a_{5}-2805 a_{2} a_{4}+231 a^{2} a_{3}\right. \\
& \left.-1650 a_{3}^{2}+924 a a_{1} a_{2}+231 a_{1}^{3}-a^{4}\right) x^{14}
\end{aligned}
$$

Let $Q\left(x_{1}, x_{2}, x_{3}\right)=0$ be the equation of the osculating quartic. If we should substitute into its left member $x_{\kappa}=y_{\kappa}(\kappa=1,2,3)$, the coefficients of all powers of $x$ up to and including the thirteenth would be equal to zero, and we should have fourteen equations for the ratios of the fifteen coefficients of $Q$. The equation $Q=0$ has been obtained by the author in this way. The details of that solution, however, will not be given here, as the equation of the osculating quartic may be obtained more easily and in a more satisfactory form by another method. We wish, however, to emphasize the fact that the equations obtained by the two different methods have been compared, for the sake of checking the results presented here, and have been found to agree.

By combining $y_{1}, y_{2}, y_{3}$ so as to eliminate the terms in $x$ up to the fourth and eighth orders, inclusive, Professor Wilczrnski has obtained as the equations of the osculating conic and cubic respectively :*

$$
x_{2}^{2}-2 x_{1} x_{3}=0 \text {, }
$$

$$
7\left(15 P_{3} P_{3}^{(3)}-20 P_{3}^{\prime} P_{3}^{\prime \prime}-567 P_{3}^{3}\right) \Omega_{1}(x)+20\left[6 P_{3} P_{3}^{\prime \prime}-7\left(P_{3}^{\prime}\right)^{2}\right] \Omega_{2}(x)=0 \text {, }
$$

where

$$
\begin{aligned}
& \Omega_{1}(x)=5\left(x_{2}^{2}-2 x_{1} x_{3}\right)\left(P_{3}^{\prime} x_{3}-3 P_{3} x_{2}\right)+12 P_{3}^{2} x_{3}^{3}, \\
& \Omega_{2}(x)=5\left(x_{2}^{2}-2 x_{1} x_{3}\right)\left(21 P_{3} x_{1}-P_{3}^{\prime \prime} x_{3}\right)-42 P_{3}^{2} x_{2} x_{3}^{2}-14 P_{3} P_{3}^{\prime} x_{3}^{3}
\end{aligned}
$$

The curve $\Omega_{1}(x)=0$ has a special significance for our problem. It is the only cubic of the pencil of cubics having eight consecutive points in common with $C_{y}$, which has a double point at $P_{y_{0}}$, and has therefore been called the eight-pointic nodal cubic.

The work will be simplified if we introduce a system of non-homogeneous coördinates $X, Y$ defined by the following equations :

$$
t_{1}=y_{1}-\frac{a_{1}}{3 a} y_{2}+\frac{a_{1}^{2}}{18 a^{2}} y_{3},
$$

* Proj. Diff. Geom., pp. 63, 64. 


$$
\begin{aligned}
& t_{2}=\theta_{1} y_{2}-\frac{\theta_{1} a_{1}}{3 a} y_{3}, \\
& t_{3}=\theta_{1}^{2} y_{3}, \\
& X=\frac{t_{2}}{t_{1}}, \quad Y=\frac{t_{3}}{t_{1}},
\end{aligned}
$$

in which $\theta_{1}$ is an abbreviation for one of the cube roots of $-a / 20$. The new triangle of reference, given by (21), is characterized by the fact that if $Y$ be developed according to powers of $X$, the development assumes the form:

$$
Y=\frac{1}{2} X^{2}+X^{5}+\alpha_{7} X^{7}+\cdots+\alpha_{14} X^{14}+\cdots,
$$

in which all the coefficients are absolute invariants. Professor WILCZYNSKI has shown that in order to obtain the canonical form (23) of the development, the triangle of reference nust be chosen as follows : * "One vertex is a point on the curve and one side of the triangle is the tangent at this point. The second side is the line upon which are situated the three points of inflection of the eightpointic nodal cubic. The third side is the polar of the intersection of the other two with respect to the osculating conic. The numerical factors, which still remain arbitrary in a projective system of coördinates after the triangle of reference has been chosen, must be determined in such a way that the coördinates of one of the three points of inflection of the eight-pointic nodal cubic shall be $(0,-\sqrt[3]{16}, 1)$, and that the coördinates of the tangent to the cubic at this point shall be $\left(2 \sqrt[3]{16}, 3 \sqrt[3]{16^{2}}, 48\right)$." We are thus dealing with a coördinate system which has a purely projective relation to the curve $C_{y}$.

We now proceed to obtain the development (23) explicitly up to terms of the fourteenth order. In addition to the two fundamental invariants $\theta_{3}$ and $\theta_{8}$, it will be necessary to have explicit expressions for the following system of invariants of equation (10), obtained from $\theta_{3}$ and $\theta_{8}$ by the Jacobian process :

$$
\begin{array}{ll}
\theta_{12}=3 \theta_{3} \theta_{8}^{\prime}-8 \theta_{3} \theta_{3}^{\prime}, & \theta_{16}=\theta_{3} \theta_{12}^{\prime}-4 \theta_{12} \theta_{3}^{\prime}, \\
\theta_{20}=3 \theta_{3} \theta_{16}^{\prime}-16 \theta_{16} \theta_{3}^{\prime}, & \theta_{24}=3 \theta_{3} \theta_{20}^{\prime}-20 \theta_{20} \theta_{3}^{\prime}, \\
\theta_{28}=\theta_{3} \theta_{24}^{\prime}-8 \theta_{24} \theta_{3}^{\prime}, & \theta_{32}=3 \theta_{3} \theta_{28}^{\prime}-28 \theta_{28} \theta_{3}^{\prime},
\end{array}
$$

From (12), we find :

$$
\begin{aligned}
\theta_{12}= & 2 \cdot 3^{2} a^{2} a_{3}-2^{3} \cdot 3^{2} a a_{1} a_{2}+2^{3} \cdot 7 a_{1}^{3}, \\
\theta_{16}= & 2 \cdot 3^{2} a^{3} a_{4}-2^{2} \cdot 3^{3} a^{2} a_{1} a_{3}-2^{3} \cdot 3^{2} a^{2} a_{2}^{2} \cdot 2^{7} \cdot 3 a a_{1}^{2} a_{2}-2^{5} \cdot 7 a_{1}^{4}, \\
\theta_{20}= & 2 \cdot 3^{3} a^{4} a_{5}-2 \cdot 3^{2} \cdot 5^{2} a^{3} a_{1} a_{4}+2^{3} \cdot 3^{2} \cdot 31 a^{2} a_{1}^{2} a_{3}-2^{2} \cdot 3^{3} \cdot 7 a^{3} a_{2} a_{3} \\
& \quad+2^{4} \cdot 3^{3} \cdot 7 a^{2} a_{1} a_{2}^{2}-2^{9} \cdot 3 \cdot 5 a a_{1}^{3} a_{2}+2^{9} \cdot 7 a_{1}^{5},
\end{aligned}
$$

* Proj. Diff. Geom., p. 86. 
1909]

$$
\begin{aligned}
\theta_{24}=2 & \cdot 3^{4} a^{5} a_{6}-2 \cdot 3^{4} \cdot 11 a^{4} a_{1} a_{5}-2 \cdot 3^{3} \cdot 67 a^{4} a_{2} a_{4}+2 \cdot 3^{2} \cdot 647 a^{3} a_{1}^{2} a_{4} \\
& -2^{2} 3^{4} 7 a^{4} a_{3}^{2}+2^{2} \cdot 3^{5} \cdot 41 a^{3} a_{1} a_{2} a_{3}-2^{4} \cdot 3^{2} \cdot 13 \cdot 29 a^{2} a_{1}^{3} a_{3} \\
& -2^{5} \cdot 3^{4} \cdot 43 a^{2} a_{1}^{2} a_{2}^{2}+2^{12} \cdot 3^{2} \cdot 5 a a_{1}^{4} a_{2}+2^{4} \cdot 3^{4} \cdot 7 a^{3} a_{2}^{3}-2^{11} \cdot 5 \cdot 7 a_{1}^{6}, \\
\theta_{28}= & 2 \cdot 3^{4} a^{6} a_{7}-2^{2} \cdot 3^{4} \cdot 7 a^{5} a_{1} a_{6}+2 \cdot 3^{2} \cdot 7 \cdot 149 a^{4} a_{1}^{2} a_{5}-2^{3} \cdot 3^{3} \cdot 5^{2} a^{5} a_{2} a_{5} \\
& +2^{4} \cdot 3^{2} \cdot 7^{2} \cdot 11 a^{4} a_{1} a_{2} a_{4}-2 \cdot 3^{3} \cdot 151 a^{5} a_{3} a_{4}-2 \cdot 3^{2} \cdot 7 \cdot 893 a^{3} a_{1}^{3} a_{4} \\
& +2^{2} \cdot 3^{4} \cdot 151 a^{4} a_{1} a_{3}^{2}-2^{2} \cdot 3^{2} \cdot 16251 a^{3} a_{1}^{2} a_{2} a_{3}+2^{2} \cdot 3^{6} \cdot 23 a^{4} a_{2}^{2} a_{3} \\
& +2^{5} \cdot 3^{2} \cdot 7 \cdot 253 a^{2} a_{1}^{4} a_{3}+2^{6} \cdot 3^{2} \cdot 2441 a^{2} a_{1}^{3} a_{2}^{2}-2^{4} \cdot 3^{6} \cdot 23 a^{3} a_{1} a_{2}^{3} \\
& -2^{14} \cdot 3 \cdot 5 \cdot 7 a a_{1}^{5} a_{2}+2^{14} \cdot 5 \cdot 7 a_{1}^{7}, \\
\theta_{32}=2 & \cdot 3^{5} a^{7} a_{8}-2^{3} \cdot 3^{4} \cdot 13 a^{6} a_{1} a_{7}+2 \cdot 3^{3} \cdot 7 \cdot 227 a^{5} a_{1}^{2} a_{6}-2^{2} \cdot 3^{4} \cdot 71 a^{6} a_{2} a_{6} \\
& -2 \cdot 3^{2} \cdot 7 \cdot 5063 a^{4} a_{1}^{3} a_{5}+2^{2} \cdot 3^{4} \cdot 1283 a^{5} a_{1} a_{2} a_{5}-2 \cdot 3^{4} \cdot 251 a^{6} a_{3} a_{5} \\
& -2 \cdot 3^{2} \cdot 222757 a^{4} a_{1}^{2} a_{2} a_{4}+2^{2} \cdot 3^{3} \cdot 4019 a^{5} a_{2} a_{4}+2 \cdot 3^{3} \cdot 11711 a^{5} a_{1} a_{3} a_{4} \\
& -2 \cdot 3^{4} \cdot 151 a^{6} a_{4}^{2}+2 \cdot 3^{2} \cdot 7 \cdot 29111 a^{3} a_{1}^{4} a_{4}-2^{2} \cdot 3^{5} \cdot 7 \cdot 373 a^{4} a_{1}^{2} a_{3}^{2} \\
& +2^{2} \cdot 3^{5} \cdot 5 \cdot 113 a^{5} a_{2} a_{3}^{2}+2^{2} \cdot 3^{3} \cdot 23 i 707 a^{3} a_{1}^{3} a_{2} a_{3} \\
& -2^{3} \cdot 3^{4} \cdot 10799 a^{4} a_{1} a_{2}^{2} a_{3}-2^{6} \cdot 3^{2} \cdot 7 \cdot 4063 a^{2} a_{1}^{5} a_{3}-2^{7} \cdot 3^{3} \cdot 16417 a^{2} a_{1}^{4} a_{2}^{2} \\
& +2^{4} \cdot 3^{4} \cdot 13697 a^{3} a_{1}^{2} a_{2}^{3}-2^{4} \cdot 3^{7} \cdot 23 a^{4} a_{2}^{4}+2^{19} \cdot 3 \cdot 5 \cdot 7 a a_{1}^{6} a_{2}-2^{16} \cdot 5 \cdot 7^{2} a_{1}^{8} .
\end{aligned}
$$

We shall also need $\theta_{36}=3 \theta_{3} \theta_{32}^{\prime}-32 \theta_{32} \theta_{3}^{\prime}$. These Jacobians, together with $\theta_{3}$ and $\theta_{8}$, form a complete system $(\Sigma)$ of invariants of equation (10), in the sense that any rational invariant whatever, involving $\theta_{3} \equiv a$, and its derivatives, $\theta_{3}^{(i)} \equiv a_{i}(i=1,2 \ldots 9)$, may be expressed rationally in terms of the invariants of the system.* Equations (25) show that $a, a_{2}, a_{3}, \ldots, a_{9}$ may be expressed rationally in terms of the members of $\Sigma$ and of $q_{1}$. Since the coefficients $\alpha_{i}$ in the development (23) are absolute invariants, wuen $a$ and its derivatives have been replaced by the members of this system, the terms in $\alpha_{i}$ involving $a_{1}$ must in the aggregate disappear. We may therefore neglect, at the outset, all terms involving $a_{1}$; and it will be understood that terms involving $a_{1}$ have been omitted from the right hand members of all the equations which follow, up to and including (31). Equations (22) now take the simple form :

or more explicitly :

$$
X=\frac{\theta_{1} y_{2}}{y_{1}}, \quad Y=\frac{\theta_{1}^{2} y_{3}}{y_{1}}
$$

$$
\theta_{1}^{-1} X=x+\frac{3}{4 !} a x^{4}+\frac{3}{6 !} a_{2} x^{6}+\frac{3}{7 !}\left(33 a^{2}+a_{3}\right) x^{7}+\frac{3}{8 !} a_{4} x^{8}
$$

* Proj. Diff. Geom., p. 36. 


$$
\begin{aligned}
& +\frac{3}{9 !}\left(183 a a_{2}+a_{5}\right) x^{9}+\frac{3}{10 !}\left(a_{6}+273 a a_{3}+3753 a^{3}\right) x^{10} \\
& +\frac{3}{11 !}\left(a_{7}+388 a a_{4}+413 a_{2}^{2}\right) x^{11}+\frac{3}{12 !}\left(a_{8}+531 a a_{5}+1512 a_{2} a_{3}\right. \\
& \left.+60165 a^{2} a_{2}\right) x^{12}+\frac{3}{13 !}\left(a_{9}+705 a a_{6}+2586 a_{2} a_{4}\right. \\
& \left.+118377 a^{2} a_{3}+1512 a_{3}^{2}+1017441 a^{4}\right) x^{13}+\cdots \\
\theta_{1}^{-2} Y= & \frac{1}{2} x^{2}+\frac{9}{5 !} a x^{5}+\frac{15}{7 !} a_{2} x^{7}+\frac{9}{8 !}\left(2 a_{3}+53 a^{2}\right) x^{8}+\frac{21}{9 !} a_{4} x^{9} \\
& +\frac{24}{10 !}\left(a_{5}+150 a a_{2}\right) x^{10}+\frac{9}{11 !}\left(3 a_{6}+671 a a_{3}+8289 a^{3}\right) x^{11} \\
& +\frac{3}{12 !}\left(10 a_{7}+3171 a a_{4}+3570 a_{2}^{2}\right) x^{12}+\frac{3}{13 !}\left(11 a_{5}+4758 a a_{5}\right. \\
& \left.+14508 a_{2} a_{3}+497772 a^{2} a_{2}\right) x^{13}+\frac{3}{14 !}\left(12 a_{9}+6867 a a_{6}\right. \\
& \left.+27195 a_{2} a_{4}+1069173 a^{2} a_{3}+16020 a_{3}^{2}+8580087 a^{4}\right) x^{14}+\cdots
\end{aligned}
$$

Reverting the series (27), we have:

$$
\begin{aligned}
x= & \theta_{1}^{-1} X-\frac{3}{4 !} a \theta_{1}^{-4} X^{4}-\frac{3}{6 !} a_{2} \theta_{1}^{-6} X^{6}+\frac{3}{7 !}\left(72 a^{2}-a_{3}\right) \theta_{1}^{-7} X^{7} \\
& -\frac{3}{8 !} a_{4} \theta_{1}^{-8} X^{8}+\frac{3}{9 !}\left(447 a a_{2}-a_{5}\right) \theta_{1}^{-9} X^{9}+\frac{3}{10 !}\left(717 a a_{3}-23058 a^{3}\right. \\
& \left.-a_{6}\right) \theta_{1}^{-10} X^{10}+\frac{3}{11 !}\left(1097 a a_{4}+973 a_{2}^{2}-a_{7}\right) \theta_{1}^{-11} X^{11}+\frac{3}{12 !}\left(1614 a a_{5}\right. \\
& \left.+3636 a_{2} a_{3}-443691 a^{3} a_{2}-a_{8}\right) \theta_{1}^{-12} X^{12}+\frac{3}{13 !}\left(2298 a a_{6}+6423 a_{2} a_{4}\right. \\
& \left.-985815 a^{2} a_{3}+3636 a_{3}^{2}+18311440 a^{4}-a_{9}\right) \theta_{1}^{-13} X^{13}+\cdots
\end{aligned}
$$

Substituting this value of $x$ in equation (28), we find the following symbolic expression for the development (23):

$$
\begin{aligned}
Y= & \frac{1}{2} X^{2}+X^{3}-\frac{6}{7 !} a_{2} \theta_{1}^{-5} X^{7}+\frac{6}{8 !}\left(105 a^{2}-a_{3}\right) \theta_{1}^{-6} X^{8}-\frac{6}{9 !} a_{4} \theta_{1}^{-7} X^{9} \\
& +\frac{6}{10 !}\left(630 a a_{2}-a_{5}\right) \theta_{1}^{-8} X^{10}+\frac{6}{11 !}\left(990 a a_{3}-43848 a^{3}-a_{6}\right) \theta_{1}^{-9} X^{11}
\end{aligned}
$$


(30)

$$
\begin{aligned}
& +\frac{6}{12 !}\left(1485 a a_{4}+1386 a_{2}^{2}-a_{7}\right) \theta_{1}^{-10} X^{12}+\frac{6}{13 !}\left(2145 a a_{5}+5148 a_{2} a_{3}\right. \\
& \left.-806922 a^{2} a_{2}-a_{8}\right) \theta_{1}^{-11} X^{13}+\frac{6}{14 !}\left(3003 a_{6}+9009 a_{2} a_{4}\right. \\
& \left.-1741284 a^{2} a_{3}+5148 a_{3}^{2}+46540494 a^{4}-a_{9}\right) \theta_{1}^{-12} X^{14}+\cdots
\end{aligned}
$$

It remains to replace the quantities $a_{i}$ by the members of the system of invariants $\Sigma$ of the differential equation (10). Solving equations (25) for these quantities, we find :

$$
a=\theta_{3}, \quad 2 \cdot 3 \theta_{3} \cdot a_{2}=\theta_{8}, \quad 2 \cdot 3^{2} \theta_{3}^{2} \cdot a_{3}=\theta_{12},
$$

$$
\begin{aligned}
& 2 \cdot 3^{2} \theta_{3}^{3} \cdot a_{4}=\theta_{16}+2 \theta_{8}^{2}, \quad 2 \cdot 3^{3} \theta_{3}^{4} \cdot a_{5}=\theta_{20}+7 \theta_{18} \theta_{8}, \\
& 2^{2} \cdot 3^{4} \theta_{3}^{5} \cdot a_{6}=2 \theta_{24}+67 \theta_{16} \theta_{8}+2 \cdot 7 \theta_{13}^{2}+2 \cdot 5^{2} \theta_{8}^{3}, \\
& 2^{2} \cdot 3^{5} \theta_{3}^{6} \cdot a_{7}=2 \cdot 3 \theta_{28}+2^{2} \cdot 5^{2} \theta_{20} \theta_{8}+151 \theta_{16} \theta_{12}+3 \cdot 127 \theta_{12} \theta_{8}^{3},
\end{aligned}
$$

$$
\begin{aligned}
2^{2} \cdot 3^{6} \theta_{3}^{7} \cdot a_{8}=2 \cdot 3 \theta_{32} & +2 \cdot 71 \theta_{24} \theta_{8}+2 \cdot 3 \cdot 5^{2} \cdot 17 \theta_{16} \theta_{8}^{2}+251 \theta_{20} \theta_{12} \\
& +3 \cdot 151 \theta_{16}^{2}+2^{5} \cdot 3 \cdot 11 \theta_{12}^{2} \theta_{8}+2 \cdot 3 \cdot 5^{2} \cdot 7 \theta_{8}^{4}, \\
2^{2} \cdot 3^{7} \theta_{3}^{8} \cdot a_{9}=2 \cdot 3 \theta_{36} & +2 \cdot 3 \cdot 97 \theta_{28} \theta_{8}+3 \cdot 131 \theta_{21} \theta_{12}+3 \cdot 7 \cdot 79 \theta_{20} \theta_{16} \\
& +2 \cdot 5^{2} \cdot 103 \theta_{20} \theta_{8}^{2}+2 \cdot 7681 \theta_{16} \theta_{12} \theta_{8} \\
& +2^{3} \cdot 3 \cdot 11 \theta_{12}^{3}+2 \cdot 3 \cdot 2351 \theta_{13} \theta_{8}^{3},
\end{aligned}
$$

where we still neglect to write the terms involving $a_{1}$. The required coefficients $\alpha_{i}$ are therefore the following absolute invariants:

$$
\begin{aligned}
& a_{7}=2^{-2} \cdot 3^{-2} \cdot 7^{-1} \theta_{3}^{-2} \theta_{1}^{-2} \theta_{8} \text {, } \\
& \alpha_{8}=-2^{-3} \cdot 3^{-3} \cdot 5 \cdot 7^{-1} \theta_{3}^{-4}\left(\theta_{12}-2 \cdot 3^{3} \cdot 5 \cdot 7 \theta_{3}^{4}\right) \text {, } \\
& a_{9}=-2^{-3} \cdot 3^{-5} \cdot 5 \cdot 7^{-1} \theta_{3}^{-5} \theta_{1}^{-1}\left(\theta_{16}+2 \theta_{8}^{2}\right) \text {, }
\end{aligned}
$$

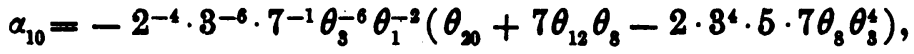

$$
\begin{aligned}
& a_{11}=2^{-3} \cdot 3^{-7} \cdot 5 \cdot 7^{-1} \cdot 11^{-1} \theta_{3}^{-8}\left(2 \theta_{24}+67 \theta_{16} \theta_{8}+2 \cdot 7 \theta_{18}^{2}-2^{2} \cdot 3^{4} \cdot 5 \cdot 11 \theta_{12} \theta_{3}^{4}\right. \\
& \left.+2 \cdot 5^{2} \theta_{8}^{3}+2^{3} \cdot 3^{7} \cdot 7 \cdot 29 \theta_{3}^{8}\right) \text {, } \\
& \alpha_{12}=2^{-5} \cdot 3^{-9} \cdot 5 \cdot 7^{-1} \cdot 11^{-1} \theta_{3}^{-9} \theta_{1}^{-1}\left(2 \cdot 3 \theta_{28}+2^{2} \cdot 5^{2} \theta_{20} \theta_{8}+151 \theta_{16} \theta_{12}\right. \\
& \left.+3 \cdot 127 \theta_{12} \theta_{8}^{2}-2 \cdot 3^{6} \cdot 5 \cdot 11 \theta_{16} \theta_{3}^{4}-2 \cdot 3^{5} \cdot 11 \cdot 37 \theta_{8}^{2} \theta_{3}^{4}\right) \\
& \alpha_{13}=2^{-5} \cdot 8^{-10} \cdot 5 \cdot 7^{-1} \cdot 11^{-1} 18^{-1} \theta_{8}^{-10} \theta_{1}^{-2}\left(2 \cdot 3 \theta_{82}+2 \cdot 71 \theta_{21} \theta_{8}+251 \theta_{20} \theta_{12}\right. \\
& -2 \cdot 8^{4} \cdot 5 \cdot 11 \cdot 13 \theta_{20} \theta_{3}^{4}+3 \cdot 161 \theta_{16}^{2}+2 \cdot 3 \cdot 5^{2} \cdot 17 \theta_{16} \theta_{8}^{2}+2^{3} \cdot 3 \cdot 11 \theta_{12}^{2} \theta_{8} \\
& \left.-2 \cdot 3^{4} \cdot 11 \cdot 18 \cdot 41 \theta_{18} \theta_{8} \theta_{8}^{4}+2 \cdot 8 \cdot 5^{2} \cdot 7 \theta_{8}^{4}+2^{2} \cdot 3^{9} \cdot 17 \cdot 293 \theta_{8} \theta_{3}^{8}\right) \text {, }
\end{aligned}
$$




$$
\begin{aligned}
\alpha_{14}= & -2^{-4} \cdot 3^{-11} \cdot 5^{2} \cdot 7^{-2} \cdot 11^{-1} \cdot 13^{-1} \theta_{3}^{-12}\left(2 \cdot 3 \theta_{36}+2 \cdot 3 \cdot 97 \theta_{28} \theta_{8}+3 \cdot 131 \theta_{24} \theta_{12}\right. \\
& -2 \cdot 3^{4} \cdot 7 \cdot 11 \cdot 13 \theta_{24} \theta_{3}^{4}+3 \cdot 7 \cdot 79 \theta_{20} \theta_{16}+2 \cdot 5^{2} \cdot 103 \theta_{20} \theta_{8}^{2} \\
& +2 \cdot 7681 \theta_{16} \theta_{12} \theta_{8}-2^{2} \cdot 3^{4} \cdot 7 \cdot 11 \cdot 13 \cdot 19 \theta_{16} \theta_{8} \theta_{3}^{4}+2^{5} \cdot 3 \cdot 11 \theta_{12}^{3} \\
& -2 \cdot 3^{4} \cdot 5 \cdot 11^{2} \cdot 13 \theta_{12}^{2} \theta_{3}^{4}+2^{3} \cdot 3^{8} \cdot 16123 \theta_{12} \theta_{3}^{8}+2 \cdot 3 \cdot 2351 \theta_{12} \theta_{8}^{3} \\
& \left.-2^{2} \cdot 3^{4} \cdot 7 \cdot 11 \cdot 13 \cdot 17 \theta_{8}^{3} \theta_{3}^{4}-2^{3} \cdot 3^{11} \cdot 7^{2} \cdot 11 \cdot 13 \cdot 41 \theta_{3}^{12}\right) .
\end{aligned}
$$

Let the equation of the osculating quartic be assumed in the form :

$$
\begin{aligned}
Q_{Y}= & \delta_{3333} Y^{4}+\delta_{3332} Y^{3} X+\delta_{3322} Y^{2} X^{2}+\delta_{3222} Y X^{3}+\delta_{2222} X^{4}+\delta_{333} Y^{3}+\delta_{332} Y^{2} X \\
& +\delta_{322} Y X^{2}+\delta_{222} X^{3}+\delta_{33} Y^{2}+\delta_{32} Y X+\delta_{22} X^{2}+\delta_{3} Y+\delta_{2} X+\delta=0
\end{aligned}
$$

If we substitute the development (23) for $Y$ into the left member of this equation, the coefficients of all powers of $X$ up to and including the thirteenth must be equal to zero, that is, we must have :

$$
\begin{aligned}
& \delta=\delta_{2}=0, \quad \delta_{22}+\frac{1}{2} \delta_{3}=0, \quad \delta_{222}+\frac{1}{2} \delta_{32}=0, \quad \frac{1}{4} \delta_{33}+\frac{1}{2} \delta_{322}+\delta_{2222}=0, \\
& \delta_{32}+\frac{1}{8} \delta_{333}+\frac{1}{4} \delta_{3322}=0, \quad \delta_{3}+\frac{1}{4} \delta_{332}+\frac{1}{2} \delta_{3222}=0 \text {, } \\
& \alpha_{7} \delta_{3}+\delta_{33}+\delta_{322}+\frac{1}{8} \delta_{3332}=0, \quad \alpha_{8} \delta_{3}+\alpha_{7} \delta_{32}+\delta_{332}+\delta_{3222}+\frac{1}{16} \delta_{3333}=0 \text {, } \\
& \alpha_{9} \delta_{3}+\alpha_{8} \delta_{32}+\alpha_{7} \delta_{33}+\alpha_{7} \delta_{322}+\frac{3}{4} \delta_{333}+\delta_{3322}=0 \text {, } \\
& \alpha_{10} \delta_{3}+\alpha_{9} \delta_{32}+\left(1+\alpha_{8}\right) \delta_{33}+\alpha_{8} \delta_{322}+\alpha_{7} \delta_{332}+\alpha_{7} \delta_{3222}+\frac{3}{4} \delta_{3332}=0 \text {, } \\
& \alpha_{11} \delta_{3}+\alpha_{10} \delta_{32}+\alpha_{9} \delta_{33}+\alpha_{9} \delta_{322}+\left(1+\alpha_{8}\right) \delta_{332}+\alpha_{8} \delta_{3222}+\alpha_{7} \delta_{3322}+\frac{3}{4} \alpha_{7} \delta_{333}+\frac{1}{2} \delta_{3333}=0 \text {, } \\
& \alpha_{12} \delta_{3}+\alpha_{11} \delta_{32}+\left(\alpha_{10}+2 \alpha_{7}\right) \delta_{33}+\alpha_{10} \delta_{322}+\alpha_{9} \delta_{332}+\left(\frac{3}{4} \alpha_{8}+\frac{8}{2}\right) \delta_{333} \\
& +\alpha_{9} \delta_{3222}+\left(1+\alpha_{8}\right) \delta_{3522}+\frac{3}{4} \alpha_{7} \delta_{3332}=0 \text {, } \\
& \alpha_{13} \delta_{3}+\alpha_{12} \delta_{32}+\left(\alpha_{11}+2 \alpha_{8}\right) \delta_{33}+\alpha_{11} \delta_{322}+\left(\alpha_{10}+2 \alpha_{7}\right) \delta_{332}+\frac{8}{4} \alpha_{9} \delta_{333} \\
& +\alpha_{10} \delta_{3222}+\alpha_{9} \delta_{3322}+\left(\frac{8}{4} \alpha_{8}+\frac{8}{2}\right) \delta_{3332}+\frac{1}{2} \alpha_{7} \delta_{3333}=0 \text {. }
\end{aligned}
$$

Solving these equations, we find:

$$
\delta_{23}=-2\left|\begin{array}{lll}
d_{11} & d_{21} & d_{31} \\
d_{12} & d_{22} & d_{32} \\
d_{13} & d_{23} & d_{33}
\end{array}\right|, \quad \delta_{222}=2\left|\begin{array}{lll}
d_{11} & d_{21} & d_{41} \\
d_{12} & d_{22} & d_{42} \\
d_{13} & d_{23} & d_{43}
\end{array}\right|,
$$

(35) $\delta_{33}-4 \delta_{2223}=8\left|\begin{array}{lll}d_{11} & d_{31} & d_{41} \\ d_{12} & d_{32} & d_{42} \\ d_{13} & d_{33} & d_{43}\end{array}\right|, \quad \Delta_{2223}=2\left|\begin{array}{lll}d_{21} & d_{31} & d_{41} \\ d_{22} & d_{32} & d_{42} \\ d_{23} & d_{33} & d_{43}\end{array}\right|$, 
where

$$
\delta_{2222}=\left|\begin{array}{cccc}
a_{7} & \alpha_{8}-4 & a_{9} & a_{10} \\
d_{11} & d_{21} & d_{31} & d_{41} \\
d_{12} & d_{22} & d_{32} & d_{12} \\
d_{13} & d_{23} & d_{33} & d_{43}
\end{array}\right|, \quad \delta_{332}=-4 \Delta_{2222}-32 \delta_{22},
$$

$$
\begin{array}{ll}
d_{11}=\alpha_{8}-6, & d_{31}=\alpha_{10}-\alpha_{8} \alpha_{7}-8 a_{7}, \\
d_{12}=\alpha_{9}-2 \alpha_{7}^{2}, & d_{32}=\alpha_{11}-2 \alpha_{9} \alpha_{7}-\alpha_{8}^{2}-4 \alpha_{8}+12, \\
d_{13}=\alpha_{10}-2 \alpha_{8} \alpha_{7}-4 \alpha_{7}, & d_{33}=\alpha_{12}-3 \alpha_{8} \alpha_{9}-8 \alpha_{7}^{2}, \\
d_{21}=\alpha_{9}-\alpha_{7}^{2}, & d_{41}=\alpha_{11}-\alpha_{9} \alpha_{7}-2 \alpha_{8}-32, \\
d_{22}=\alpha_{10}-3 \alpha_{8} \alpha_{7}+2 \alpha_{7}, & d_{12}=\alpha_{12}-2 \alpha_{10} \alpha_{7}-\alpha_{9} \alpha_{8}+2 \alpha_{9}-6 \alpha_{7}^{2}, \\
d_{23}=\alpha_{11}-\alpha_{9} \alpha_{7}-2 \alpha_{8}^{2}+6 \alpha_{8}-12, & d_{43}=\alpha_{13}+6 \alpha_{10}-2 \alpha_{10} \alpha_{8}-\alpha_{9}^{2}-14 \alpha_{8} \alpha_{7}-28 \alpha_{7} ;
\end{array}
$$

so that the equation of the osculating quartic is

$$
\begin{aligned}
& \left(\delta_{33}-4 \delta_{2223}\right)\left(2 Y-X^{2}-8 X Y^{2}\right) Y-\delta_{332}\left(X^{3}+16 Y^{3}-2 X Y\right) Y \\
& \quad+2 \delta_{2222}\left(X^{2}-2 Y\right)^{2}+2 \delta_{223}\left(X^{2}-2 Y\right) X+2 \delta_{22}\left(X^{2}-2 Y\right) \\
& \quad+\left[2 \alpha_{7}\left(\delta_{33}-4 \delta_{2222}\right)-8 a_{9} \delta_{22}-8\left(\alpha_{8}-4\right) \delta_{222}\right]\left(X^{2}-2 Y\right) Y^{2} \\
& \quad+64\left[\left(\alpha_{8}-2\right) \delta_{22}+a_{7} \delta_{222}\right] Y^{4}+8\left(4 \alpha_{7} \delta_{22} Y^{2}+2 \delta_{222} X Y+\delta_{22} X^{2}\right) X Y=0,
\end{aligned}
$$

where all of the coefficients are absolute invariants. This is easily reduced to homogeneous form, if desired, by equations (22). Referred to the same triangle, the equations of the osculating conic and cubic are respectively:

$$
\begin{gathered}
X^{2}-2 Y=0 \\
\alpha_{7}\left(2 Y-X^{2}-8 X Y^{2}\right)+2 \alpha_{7}^{2}\left(X^{2}-2 Y\right) Y \\
+\left(\alpha_{8}-4\right)\left(X^{3}+16 Y^{3}-2 X Y\right)=0 .
\end{gathered}
$$

When $\alpha_{7}=0$, (39) reduces to the equation of the eight-pointic nodal cubic.

If we substitute the canonical development (23) of the curve $C_{y}$ into the left member of the equation (33) of the osculating quartic, we find that the coefficient of $X^{14}$ is

$$
\begin{aligned}
a_{14} \delta_{3}+\alpha_{13} \delta_{32}+\left(\alpha_{12}+2 \alpha_{9}+\alpha_{7}^{2}\right) \delta_{33}+\alpha_{12} \delta_{322}+\left(\alpha_{11}+2 \alpha_{8}\right) \delta_{338}+\left(\frac{3}{4} a_{10}+3 \alpha_{7}\right) \delta_{333} \\
+\alpha_{11} \delta_{3223}+\left(a_{10}+2 \alpha_{7}\right) \delta_{3523}+\frac{8}{4} \alpha_{9} \delta_{3352}+\left(\frac{1}{2} \alpha_{8}+\frac{8}{2}\right) \delta_{3359}
\end{aligned}
$$

When this expression vanishes, the osculating quartic has fifteen consecutive 
points in common with $C_{y}$ at $P_{y_{0}}$ or, as we may say, hyperosculates $C_{y}$ at that point. This condition may be put into the form:

where

$$
K=\left|\begin{array}{llll}
d_{11} & d_{21} & d_{31} & d_{41} \\
d_{12} & d_{22} & d_{32} & d_{42} \\
d_{13} & d_{23} & d_{33} & d_{43} \\
d_{14} & d_{24} & d_{34} & d_{44}
\end{array}\right|=0
$$

$$
\begin{aligned}
& d_{14}=\alpha_{11}-2 \alpha_{9} \alpha_{9}-4 \alpha_{8}-\alpha_{7}^{3}-24, \\
& d_{24}=\alpha_{12}-\alpha_{10} \alpha_{7}-2 \alpha_{9} \alpha_{8}+6 \alpha_{9}-\alpha_{8} \alpha_{7}^{2}-2 \alpha_{7}^{2}, \\
& d_{34}=\alpha_{13}-\alpha_{10} \alpha_{8}-2 \alpha_{9}^{2}-\alpha_{9} \alpha_{7}^{2}-16 \alpha_{8} \alpha_{7}, \\
& d_{44}=\alpha_{14}+6 \alpha_{11}-3 \alpha_{10} \alpha_{9}-14 \alpha_{9} \alpha_{7}-\alpha_{10} \alpha_{7}^{2}-8 \alpha_{8}^{2}-40 \alpha_{8}-144 .
\end{aligned}
$$

If the invariant equation (40) is satisfied for all values of $x$, that is, at all points of the curve $C_{y}$, this curve is itself a quartic.

UNIVIRSITY OF ILLINOIS,

February 12, 1909. 